\title{
What is Specialized Care in Status Epilepticus and in Which ICU?
}

\author{
Sophie Demeret $\cdot$ Nicolas Weiss $\cdot$ Francis Bolgert $\cdot$ \\ Vincent Navarro
}

Published online: 29 May 2013

(c) Springer Science+Business Media New York 2013

Status epilepticus (SE) is a serious, potentially life-threatening condition. The outcome of SE is variable, while some patients may present neurological deficits, others recover without sequellae. The reasons for these different outcomes are not fully understood, but some prognostic factors have been identified, such as the age, the clinical type of SE, a previous history of epilepsy, the etiology, and the delay of treatment onset [1-3].

S. Demeret $\cdot$ N. Weiss $\cdot$ F. Bolgert

Neurological Intensive Care Unit, Neurology Department, Groupe Hospitalier Pitié-Salpêtrière, Assistance PubliqueHôpitaux de Paris and Université Pierre et Marie Curie Paris 6, Paris, France

S. Demeret · N. Weiss $\cdot$ F. Bolgert · V. Navarro Institut Hospitalo-Universitaire-Institut de Neurosciences Translationnelles de Paris, Groupe Hospitalier Pitié-Salpêtrière, Assistance Publique-Hôpitaux de Paris and Université Pierre et Marie Curie Paris 6, Paris, France

\section{Navarro}

Departments of Neurophysiology and Neurology, Groupe Hospitalier Pitié-Salpêtrière, Assistance Publique-Hôpitaux de Paris and Université Pierre et Marie Curie Paris 6, Paris, France

\section{Navarro}

Cortex and Epilepsy Team, Centre de Recherche de l'Institut du Cerveau de la Moelle (INSERM UMRS795-CNRS UMR

7225-UPMC), ICM, Paris, France

\section{N. Weiss $(\bowtie)$}

Neurological Intensive Care Unit, Neurology Department, La Pitié-Salpêtrière Hospital, Assistance Publique-Hôpitaux de Paris and Université Pierre et Marie Curie Paris 6, Paris, France e-mail: nicolas.weiss@psl.aphp.fr
Despite being an important stage in the treatment, there have been very few studies on the various management options when patients are admitted in intensive care.

In this retrospective study, Valeras et al. compared the management and outcome of 168 cases of SE admitted to either a neurological intensive care unit (NICU) $(27 \%)$ or a medical intensive care unit (MICU) $(73 \%)$ in the same tertiary care hospital. The authors did not find a significant difference in terms of length of hospital stay, mortality, or outcome at discharge. This finding remained after adjustment for confounding factors. However, on admission, there were significant differences between the patient groups. Stroke accounted for $39 \%$ and toxic and metabolic etiologies for $11 \%$ of cases in the NICU group compared to 12 and $21 \%$, respectively, in the MICU group. The APACHE-II severity score at admission was higher in the MICU group than in the NICU group (17.5 vs. 13.4), as was consciousness impairment ( 48 vs. $22 \%$ ). There was no significant difference in terms of mortality, even if a trend to decreased mortality existed in NICU ( $4 \%$ in the NICU group; $8 \%$ in the MICU group) and no difference in outcome according to Rankin score. Regarding the management modalities, the only significant difference related to continuous EEG monitoring, performed in $85 \%$ of cases in NICU compared to $30 \%$ in MICU. The higher rate of intubation in MICU (72\%, compared to $48 \%$ in NICU) was partially correlated with consciousness impairment.

Should one therefore conclude from this study that there is no benefit to be gained from specialist intensive care in the management of SE, in contrast to what has been demonstrated in other acute neurological pathologies $[4,5]$ ?

We are convinced that some SEs may benefit from a neurological ICU, even if the study of Varelas et al. failed 
to demonstrate any superiority. The main reason of this failure lies in the heterogeneity of SE, which makes it difficult to properly analyze prognostic factors.

The etiology of the SE is itself a major prognostic factor [3]. In the case of acute brain injury, the prognosis is principally that of the underlying cause, even if the SE can be an aggravating factor. Apart from these acute symptomatic SEs, many SEs are a severe epileptic manifestation arising from a scar or chronic brain lesion, in patients who are often already on antiepileptic medication. In these remote symptomatic cases, the prognosis is far better [6] and is directly related to the SE and its management. The significant differences in etiologies between the two groups, in the Varelas study, may well have blurred the impact of specialized management.

The type of seizures, namely whether they are convulsive or non-convulsive, partial or generalized, will also have an impact on the prognosis. Generalized convulsive SEs are associated with numerous extracerebral factors having a worsening effect on neuronal metabolism [7]. In the other clinical types of SE, the excito-toxicity related to continuous discharges is variable, depending on many factors: some are unknown; others can be related to the brain structures involved or the existence or not of a previous history of epilepsy [8].

What is the place and expected benefits of a specialized unit to manage SE?

(1) Dispatching SE to NICU or MICU can be based on the type and severity of the status.

In the majority of cases, SE stops after the first dose of benzodiazepines and invasive intensive care is not required $[9,10]$.

In the case of refractory SE, namely when seizures did not respond to two antiepileptic drugs, most patients will have been placed under general anesthetic and mechanical ventilation. The anesthetic weaning is often easy, without any recurrence of the status, and its management may not significantly benefit from a neurological team.

Patients with several organ dysfunctions, furthermore if they are possible candidates for specific techniques, e.g., renal replacement therapy, extracorporeal life support, should be admitted in a MICU.

Management of partial SE by a neurointensivist with antiepileptic drugs could avoid general anesthesia as suggested by Varelas et al. who found that fewer patients were intubated in the NICU.

Few cases of SE are superrefractory, with persistence or recurrence of status, whether clinical or based on EEG evidence. The management of anesthetic and antiepileptic drugs of these superrefractory SEs requires a multidisciplinary team of neurointensivists, neurologists, electrophysiologists, neuroradiologists, and specialist nurses.

(2) We pointed some expected benefits of the management of SE in a NICU.

NICU is associated to better chart documentation, and seizures description appears as being fundamental to both interpret EEG findings but also choose the best antiepileptic drug.

The management of general anesthesia is poorly defined, and the clinician has to balance the risk of a recurrence of seizures with the potential complications associated with prolonging sedation and mechanical ventilation $[11,12,13]$. In NICU, the use and duration of anesthesia may be limited, notably to treat partial seizures.

During the withdrawal of anesthetic agents, isolated epileptic seizures may occur, especially if the SE lasted for a long period; it is important to deal with these short seizures appropriately, moving from the management of SE to the management of epilepsy, which requires a different approach. This step requires the experience of neurointensivists, neurologists, and epileptologists used to dealing with forms of epilepsy other than SE.

Continuous EEG monitoring allows early detection of seizures recurrence, and real-time anesthetic medication adjustment. This type of monitoring is principally available in NICU, where the medical and paramedical staff is experienced in its use. This also involves a close collaboration with the neurophysiological staff. However, data on the usefulness of cEEG are still lacking in SE [14].

In comatose patients, the EEG can be difficult to interpret and diagnostic errors are common. EEG recordings in the case of severe encephalopathies or brain injuries show grapho-elements (PLEDs, triphasic potentials) that can be wrongly interpreted as resulting from SE, leading to an unwarranted increase of sedation. We therefore reported the observation of 10 patients referred to our NICU as refractory nonconvulsive status, whose final diagnosis was sporadic Creutzfeldt-Jakob disease with EEG features mimicking a status [15]. In particular, the test using intravenous injection of a benzodiazepine is suggestive of an epileptic origin only if both clinical and EEG symptoms improve. The combined expertise of a clinical neurologist trained in epileptology and an electrophysiologist trained in intensive care recordings is essential to establish the correct diagnosis.

Less common etiologies of SE can be difficult to diagnose and may pose therapeutic problems. SE can be a complication in many congenital epileptic 
diseases, like Dravet syndrome; the clinical semiology, EEG and treatment are very different, and epileptologist's expertise is crucial [16]. It is important to be aware not to use aggressive treatment in the form of general anesthesia for myoclonic SEs, which may have a spontaneously favorable course, and to suspect a mitochondrial disease, which would contraindicate the use of sodium valproate [17]. Similarly, tonic SE, as observed in the Lennox-Gastaut syndrome can be worsened by benzodiazepine. SE is a classic complication of infectious or inflammatory encephalitis. Early etiological diagnosis is difficult in some rare cases and requires a specialized team, aware of the newly described syndromes related to antibodies against neuropils [18]. The ability to distinguish clonic movements of epileptic origin from other abnormal involuntary movements of brainstem or basal ganglia origin, such as those seen in anti-NMDAr encephalitis, is of paramount importance for the therapeutic management [19]. The rapid introduction of corticosteroid or immunomodulatory drugs, without waiting for confirmation of the etiology, requires experience of these pathologies.

Further studies are now needed to determine the most suitable management modalities for SE patients on arrival in intensive care, especially in term of depth and duration of general anesthesia. NICU management could enable easier research on SE.

As recently discussed during the 4th Colloquium on Status Epilepticus, held in Salzburg, SE regroups heterogeneous conditions, requiring a precise, positive, and syndromic diagnosis, based on clinical, EEG, and imaging features. Their management must be tailored to the specific entities. The challenge for the NICU in the next few years will be the identification of SEs requiring aggressive management, and those for which such aggressive strategy must be avoided.

\section{References}

1. Logroscino G, Hesdorffer DC, Cascino G, Annegers JF, Hauser WA. Short-term mortality after a first episode of status epilepticus. Epilepsia. 1997;38:1344-9.

2. DeLorenzo RJ, Hauser WA, Towne AR, Boggs JG, Pellock JM, Penberthy L, et al. A prospective, population-based epidemiologic study of status epilepticus in Richmond, Virginia. Neurology. 1996;46:1029-35.

3. Neligan A, Shorvon SD. Frequency and prognosis of convulsive status epilepticus of different causes. A systematic review. Arch Neurol. 2010;67:831-40.

4. Knopf L, Staff I, Gomes J, McCullough L. Impact of a neurointensivist on outcomes in critically ill stroke patients. Neurocrit Care. 2012;16:63-71.

5. Varelas PN, Eastwood D, Yun HJ, et al. Impact of a neurointensivist on outcomes in patients with head trauma treated in a neurosciences intensive care unit. J Neurosurg. 2006;104:713-9.

6. Towne AR, Pellock JM, Ko D, DeLorenzo RJ. Determinants of mortality in status epilepticus. Epilepsia. 1994;35:27-34.

7. Walton NT. Systemic effects of generalized convulsive status epilepticus. Epilepsia. 1993;34:S54-8.

8. Chen JW, Naylor DE, Wasterlain CG. Advances in pathophysiology of status epilepticus. Acta Neurol Scand Suppl. 2007;186: $7-15$.

9. Alldredge BK, Gelb AM, Isaacs SM, Corry MD, Allen F, Ulrich S, Gottwald MD, O’Neil N, Neuhaus JM, Segal MR, Lowenstein DH. A comparison of lorazepam, diazepam, and placebo for the treatment of out-of-hospital status epilepticus. N Engl J Med. 2001;345:631-7.

10. Silbergleit R, Durkalski V, Lowenstein D, Conwit R, Pancioli A, Palesch Y, Barsan W, NETT Investigators. Intramuscular versus intravenous therapy for prehospital status epilepticus. N Engl J Med. 2012;366:591-600.

11. Hocker SE, Britton JW, Mandrekar JN, Wijdicks EF, Rabinstein AA. Predictors of outcome in refractory status epilepticus. JAMA Neurol. 2013;70:72-7.

12. Iyer VN, Hoel R, Rabinstein AA. Propofol infusion syndrome in patients with refractory status epilepticus: an 11-year clinical experience. Crit Care Med. 2009;37:3024-30.

13. Miller MA, Forni A, Yogaratnam D. Propylene glycol-induced lactic acidosis in a patient receiving continuous infusion pentobarbital. Ann Pharmacother. 2008;42:1502-6.

14. Sutter R, Stevens RD, Kaplan PW. Continuous electroencephalographic monitoring in critically ill patients: indications, limitations, and strategies. Crit Care Med. 2013;41:1124-32.

15. Lapergue B, Demeret S, Denys V, Laplanche JL, Galanaud D, Verny M, Sazdovitch V, Baulac M, Haïk S, Hauw JJ, Bolgert F, Brandel JP, Navarro V. Sporadic Creutzfeldt-Jakob disease mimicking nonconvulsive status epilepticus. Neurology. 2010;74: 1995-9.

16. Dulac O, Takahashi T. Status epilepticus. Handb Clin Neurol. 2013;111:681-9.

17. Lin CM, Thajeb P. Valproic acid aggravates epilepsy due to MELAS in a patient with an A3243G mutation of mitochondrial DNA. Metab Brain Dis. 2007;22:105-9.

18. Dalmau J. Status epilepticus due to paraneoplastic and non paraneoplastic encephalitides. Epilepsia. 2009;50(Suppl 12):58-60.

19. Baizabal-Carvallo JF, Stocco A, Muscal E, Jankovic J. The spectrum of movement disorders in children with anti-NMDA receptor encephalitis. Mov Disord. 2013;28(4):543-7. 